\title{
The Zadko Telescope: Exploring the Transient Universe
}

D. M. Coward ${ }^{1,11}$, B. Gendre ${ }^{2,3}$, P. Tanga ${ }^{4}$, D. Turpin ${ }^{5,6}$, J. Zadko $^{1}$, R. Dodson ${ }^{7}$, M. Devogéle ${ }^{8,9}$, E. J. Howell ${ }^{1}$, J. A. Kennewell ${ }^{1}$, M. Boër ${ }^{3}$, A. Klotz ${ }^{5,6}$, D. Dornic ${ }^{10}$, J. A. Moore ${ }^{1}$ and A. Heary ${ }^{1}$

${ }^{1}$ School of Physics, University of Western Australia, M013, Crawley WA 6009, Australia

${ }^{2}$ University of the Virgin Islands, John Brewers Bay, St Thomas, U.S. Virgin Islands 00802-9990, USA

${ }^{3}$ CNRS - ARTEMIS, boulevard de l'Observatoire, CS 34229, 06304 Nice Cedex 04, France

${ }^{4}$ Boulevard de l'Observatoire, CS 34229, 06304 Nice Cedex 04, France

${ }^{5}$ Université de Toulouse; UPS-OMP; IRAP; Toulouse, France

${ }^{6}$ CNRS; IRAP; 14, avenue Edouard Belin, F-31400 Toulouse, France

${ }^{7}$ International Centre for Radio Astronomy Research, M468, The University of Western Australia, Crawley, WA 6009, Australia

${ }^{8}$ Laboratoire Lagrange, Université Côte d'Azur, Observatoire de la Côte d'Azur, CNRS, Blvd. de 1'Observatoire, CS 34229, 06304 Nice cedex 4, France

${ }^{9}$ Institut d'Astrophysique, Géophysique et Océanographie, Université de Liège, Belgium

${ }^{10}$ Aix Marseille Université, CNRS/IN2P3, CPPM UMR 7346, 13288, Marseille, France

${ }^{11}$ Email: david.coward@uwa.edu.au

(RECEIVED November 16, 2016; ACCEPTED November 25, 2016)

\begin{abstract}
The Zadko telescope is a $1 \mathrm{~m} \mathrm{f} / 4$ Cassegrain telescope, situated in the state of Western Australia about 80-km north of Perth. The facility plays a niche role in Australian astronomy, as it is the only meter class facility in Australia dedicated to automated follow-up imaging of alerts or triggers received from different external instruments/detectors spanning the entire electromagnetic spectrum. Furthermore, the location of the facility at a longitude not covered by other meter class facilities provides an important resource for time critical projects. This paper reviews the status of the Zadko facility and science projects since it began robotic operations in March 2010. We report on major upgrades to the infrastructure and equipment (2012-2014) that has resulted in significantly improved robotic operations. Second, we review the core science projects, which include automated rapid follow-up of gamma ray burst (GRB) optical afterglows, imaging of neutrino counterpart candidates from the ANTARES neutrino observatory, photometry of rare (Barbarian) asteroids, supernovae searches in nearby galaxies. Finally, we discuss participation in newly commencing international projects, including the optical follow-up of gravitational wave (GW) candidates from the United States and European GW observatory network and present first tests for very low latency follow-up of fast radio bursts. In the context of these projects, we outline plans for a future upgrade that will optimise the facility for alert triggered imaging from the radio, optical, high-energy, neutrino, and GW bands.
\end{abstract}

Keywords: gravitational waves, instrumentation: miscellaneous, minor planets, asteroids: general, (stars:) gamma-ray burst: general

\section{INTRODUCTION}

\subsection{Transient source astronomy}

The Universe is teeming with fleeting transients, seen across the entire electromagnetic (EM) spectrum. In the optical, some last for only a few seconds, whereas others change slowly in brightness over years. Sources classed as transient include cataclysmic variable stars, blazars, stellar flares, hypernovae, and supernovae, whereas many of the recently discovered sources are not easily explained. Some transients, such as gamma ray bursts (GRBs), have complex emissions spanning the gamma, X-ray, and optical spectra, and are possibly strong gravitational wave $(\mathrm{GW})$ and neutrino sources.
The 21st century has heralded a new trans-spectral era for exploration of the Universe: For the first time humankind is able to observe the Universe across the whole EM spectrum and beyond. Space-based GRB detectors have opened a window into a Universe teeming with short-lived exotic phenomena, the so-called transient Universe. But to fully reveal the nature of transients requires near simultaneous observations from the radio, optical and high energy spectra, combined with data from the GW spectrum, initiated in late 2016 by the first observations of binary black hole mergers (Abbott et al., 2016a, 2016c; The LIGO Scientific Collaboration et al., 2016).

Multi-messenger astronomy spanning the entire EM spectrum has yet to be fully exploited because of the difficulty 
Table 1. Zadko telescope imaging specifications.

\begin{tabular}{ll}
\hline \hline CCD FOV & $23^{\prime} .6 \times 23^{\prime} .6$ \\
pixel scale & $2048 \times 204813.5 \mu \mathrm{m}$ square pixels \\
pixel size & $a$ \\
filters & $0.69 \operatorname{arcsec}$ \\
camera & SDSS u, g, r, i and clear \\
CCD & Andor iKon-L \\
mag limits & DW436-BV back-illuminated sensor \\
phot accuracy & $m \approx 21.5180 \mathrm{~s}$ exposure \\
seeing & dispersion $<0.01$ for $m=11$ \\
\hline \hline
\end{tabular}

${ }^{\text {a }}$ The CCD images employ $2 \times 2$ binning because of the seeing limitations, resulting in pixel sizes of about 1.4 arcsec.

of rapid response automation, data processing, and coordination required between independent facilities. Despite the difficulties, progress has been made: We are entering a new dawn in the discovery and study of exotic transients. Space-based satellite detectors are being networked to optical telescopes for rapid follow-up imaging of transient targets. Furthermore, for the first time robotic telescopes in Australia, Chile, and France are being networked together, performing automated imaging of transients analogous to a distributed parallel computing network, where imaging tasks are allocated to particular instruments. Another recent innovation is the coordination of optical and radio telescopes for coincident transient detection, which requires automated communication between different facilities to image the same sky (see Section 9.1 for details).

\section{THE ZADKO TELESCOPE}

The Zadko telescope (Coward et al., 2010) is a $1 \mathrm{~m} \mathrm{f} / 4$ Cassegrain telescope constructed by DFM Engineering Ltd., situated in the state of Western Australia at longitude, $115^{\circ} 42^{\prime}$ $49^{\prime \prime} \mathrm{E}$, latitude, $31^{\circ} 21^{\prime} 24^{\prime \prime} \mathrm{S}$, and at an altitude of $50 \mathrm{~m}$ above sea level. Situated about 70-km north of Perth (see Coward et al., 2010,for more detail). It was donated to University of Western Australia (UWA) by resource company Claire Energy, and opened by Australia's Chief Scientist on 1 April 2009 , with a science goal of exploring a previously uncharted region of the 'transient sky'. The most important imaging specifications of the Zadko telescope are outlined in Table 1.

In 2010, the telescope control system was robotized using the TAROT robotic telescope software system. This suite of independently running programs comprises two main components: AudeLA ${ }^{1}$ and Robotic Observatory Software (ROS). There is also third party software (mostly drivers) for the mechanical and sensors of the observatory (i.e. telescope + dome + dome interior + weather station). For the Zadko Telescope, The DFM supplied Telescope Control System is interfaced to ROS via the ASCOM (Astronomy Common Object Model) protocol.

A key feature of the telescope is the optimization for performing automated follow-up from alerts or triggers from

\footnotetext{
${ }^{1}$ http://www.audela.org/english_audela.php
}

different external instruments. In addition, it employs a dynamic scheduling system that allows other projects to operate when not in alert mode. Furthermore, the location of the facility at a longitude not covered by other meter class facilities provides unique access to the Southern transient sky.

The aim of this paper is to review the status of the Zadko facility and science projects since it began robotic operation. First, we will report on several major upgrades to the infrastructure and equipment that have improved the capability of the facility for transient source follow-up. Second, we review the core science projects that include:

- GRBs: Rapid optical follow-up of Swift alerts

- ANTARES neutrino alert follow-up

- Solar system studies: Gaia satellite follow-up of asteroids and photometry of 'Barbarian asteroids'

- GW follow-up of alerts from aLIGO/Virgo

- Nearby supernovae and lensed supernovae search

- Emerging high-priority project: automated low latency follow-up of radio transients (fast radio bursts)

- Education and training: space debris tracking (pilot project)

\section{ZADKO TELESCOPE INFRASTRUCTURE AND EQUIPMENT UPGRADE}

The Zadko telescope, which was first installed in June 2008, was initially housed within a $6.7-\mathrm{m}$ fibreglass dome. Due to the robotic nature of the telescope that can make hundreds of movements throughout the night, the demands placed on the fibreglass dome to continually adjust its position to accommodate these movements caused it to be in constant need of maintenance and repair and consequential failure.

In March 2011, it was decided to replace the fibreglass dome with a state-of-the-art purpose built rolling roof facility with a dedicated constant temperature climate controlled operation room. In addition to this, a climate controlled telescope Service room to mirror the Operation room was also added. This has the added benefit of allowing for possible future conversion into a second control room if further telescopes are added to the facility.

Due to the environmentally hostile and remote location of the telescope, it was decided to leave the telescope in its existing position during the decommissioning of the fibreglass dome and the construction phase of the new building. This was achieved by erecting a temporary watertight structure around the telescope before dis-assembly of the fibreglass dome.

As the majority of the new structure is steel and prefabricated off-site the installation was performed in a relatively short time frame of approximately six months. The curved corrugated zinc-alumina roof was then installed without gutters so as to prevent leaf litter build up as a fire prevention strategy. The walls and ceiling panels of the Operation, Service, and Telescope rooms are 100-mm-thick EPS-coreinsulated panel that assist in maintaining reasonably constant 
temperatures throughout the year. Both Operation and Service rooms have split system air-conditioning units installed for constant temperature control.

The main control for the automatic operation of the rolling roof is provided by a programmable logic controller (PLC)/Burgess system that was designed and installed by our French collaborators at Observatoire de Haute-Provence. This includes electrical and mechanical interfaces for temperature, humidity and wind sensors, flat field lamp, emergency stops, and limit switches plus a main control panel.

An important feature of the building design was to protect the telescope from sudden adverse weather events. This was achieved by installing an external mast fitted with real-time weather sensors (cloud, rain, humidity, wind, temp) that enables a signal to be sent to the PLC/Burgess. The rolling roof immediately responds to these signals and may close and reopen several times during the night according to the weather conditions. Approximate roof travel time to close/open is 2 min in either direction.

The main drive of the rolling roof consists of two threephase $0.75-\mathrm{kW}$ brake flange motors connected to an in-line helical gear box with a 46:1 ratio installed on either side of the structure connected to a geared rack. The motors are powered by a variable speed drive with single-phase input and three-phase output that is located within the PLC/Burgess cabinet. All critical drive components of the operation are supported by a Socomec 7-kVA uninterrupted power supply (UPS) in the event of an external power failure. These include the ability for the roof to close and for all computer systems to remain active (see Figure 1).

The existing Guide Acquired Module (GAM) that houses the Andor IKON-L camera has been redesigned to provide an improved field of view, provision for larger filters, and to allow for future spectroscopy. This new GAM will be installed in 2016. Estimated downtime is 6 weeks to allow for both primary and secondary mirrors to be removed for recoating and re-installation. A new flat field screen has been installed, suspended from the rolling roof at a distance and angle to achieve optimum results for camera calibration. Improvements to computer systems, data, and internet provision are continuous and ongoing with major areas of concern being poor internet service and intermittent power outages.

\subsection{Environmental impact on equipment}

The observatory site has provided a very challenging environment for the equipment. In particular, humidity levels often rise to above $90 \%$ during the night, mainly between June and September. Even though the system automatically shuts down at this humidity level, the primary mirror and camera (which is thermo-electrically cooled) are partially exposed. Condensation combined with dust, salt, and pollen has accelerated the degradation of the mirror coating. To reduce this, the mirror has to be cleaned regularly on the time scale of 4-6 weeks. Figure 2 shows the corrosion on the primary mirror coating and the effect of condensation on the main charge-
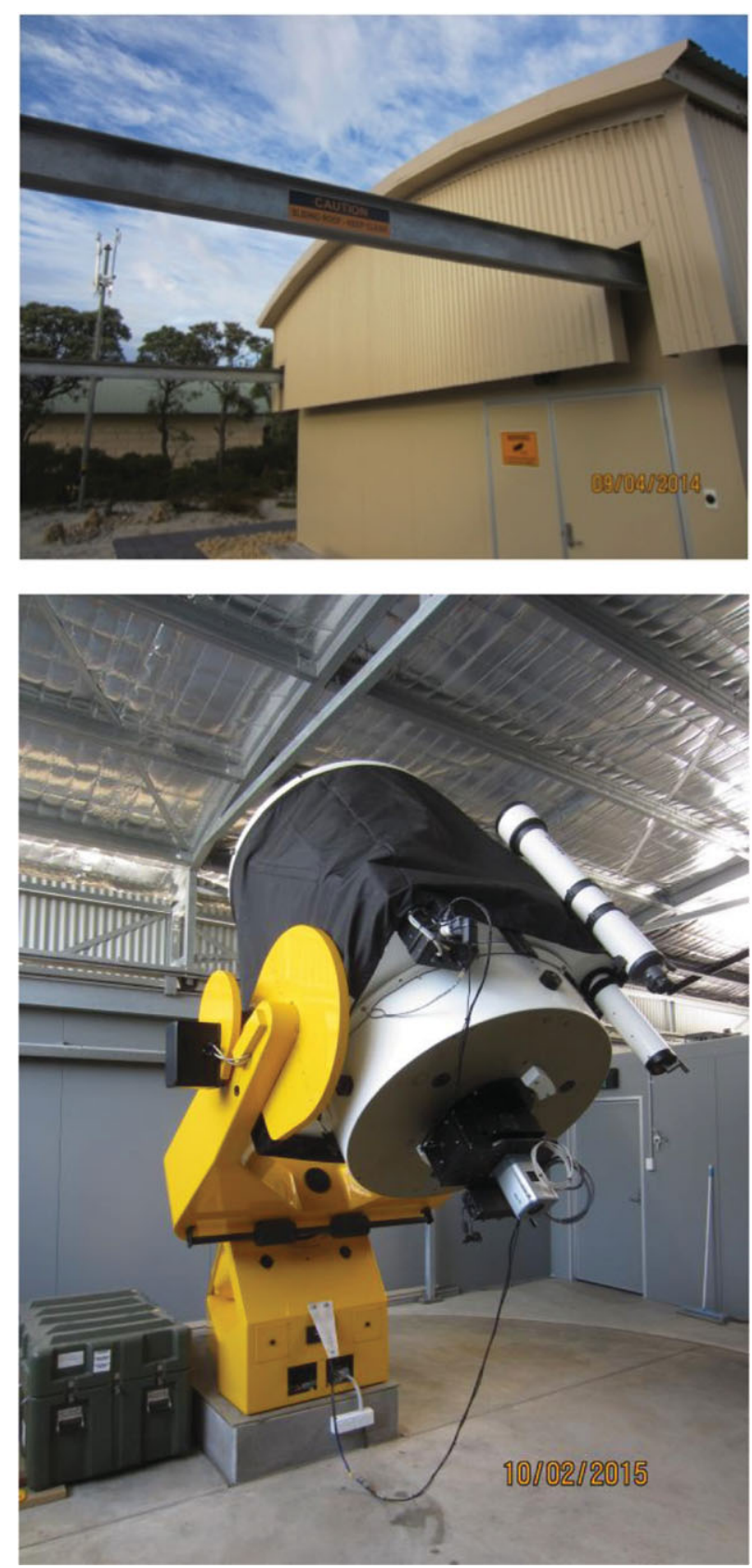

Figure 1. Top: The new observatory constructed in 2012, with key features including fully automated slide off roof controlled by weather sensors interfaced to a PLC system. Bottom: The Zadko Telescope ready for operation.

coupled device (CCD) camera circuit board. UWA technical staff are testing several filter options for the CCD that will inhibit dust intake and condensates on the circuit board.

\subsection{RAPIDO: Plans for a co-located wide field rapid response telescope}

The French leaders of the TAROT/Zadko projects have designed and constructed a prototype extremely rapid response 


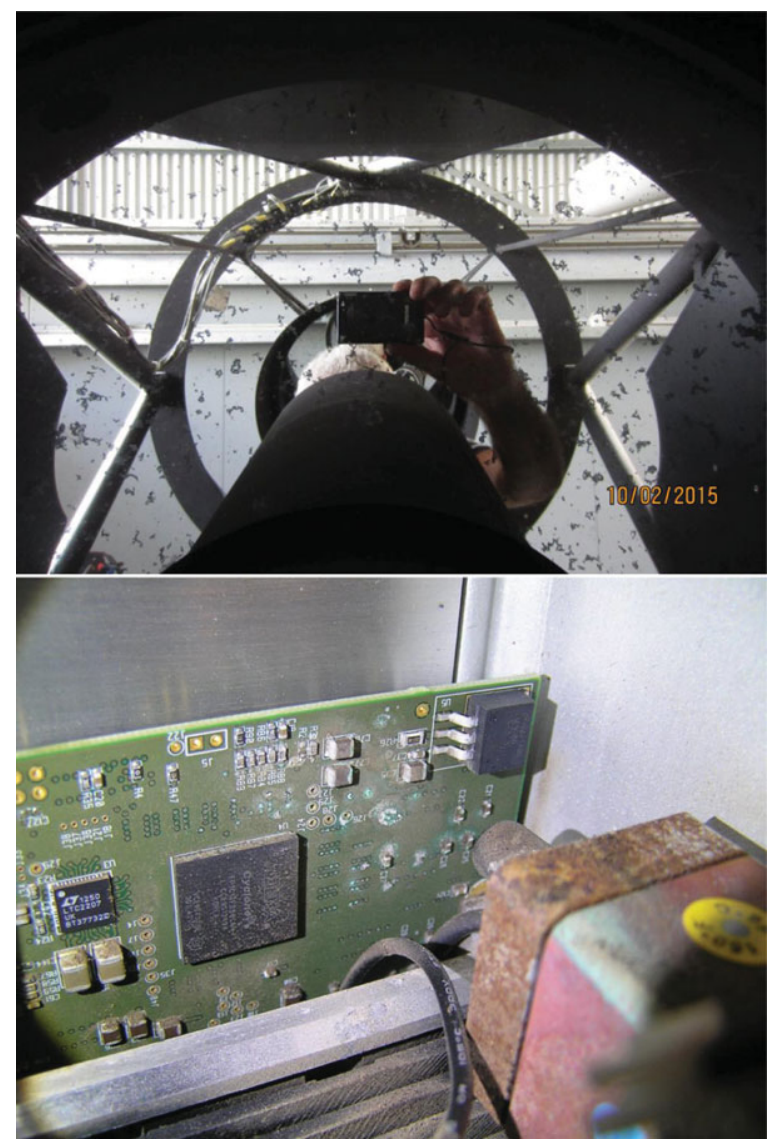

Figure 2. Top: The primary mirror, showing corrosion of the coating (grey patterns) from the high levels of humidity and condensation, combined with dust and pollen. Bottom: The main circuit board of the IKON-L CCD camera, which is exposed to the atmosphere, showing corrosion on all metallic components and connectors. This eventually led to a camera failure after about 14 months of use.

telescope mount (slew speed $45^{\circ}$ per s) ${ }^{2}$. This mount is planned to control two off-the-shelf wide-field [combined 8-10 $\mathrm{deg}^{2}$ field of view $\left.(\mathrm{FoV})\right]$ Takahashi $(2 \times 20 \mathrm{~cm})$ telescopes. The first imaging using a FLI Kaf16003 camera obtained a limiting magnitude of about 18 (clear filter). RAPIDO and Zadko will share the same robotic control system, which will enable RAPIDO to automatically send alerts to Zadko for deep follow-up of GW and fast radio burst (FRB) candidates. The instrument is upgradable to accommodate a 0.4-m telescope and will be co-located with the Zadko Telescope, subject to funding.

\section{GAMMA-RAY BURST FOLLOW-UP}

\subsection{Gamma-ray bursts}

One class of extra-galactic transient that triggers the Zadko Telescope is GRBs. They are the brightest EM explosions in the Universe (see the review by Meszaros, 2006). Usually detected first in gamma and X-rays by NASA's Swift satel-

\footnotetext{
${ }^{2}$ http://cador.obs-hp.fr/rapido/
}

lite (Gehrels et al., 2004,albeit other instruments can detect GRBs), they have distinct but poorly understood optical signals, including prompt emission, and a delayed afterglow.

The popular progenitor model for a GRB is either a massive stellar collapse, or a compact object merger triggering an explosion causing a burst of collimated gamma-rays powered by accretion onto the newly formed compact object. There are two classes of GRBs categorised by their durations and spectra: namely short-hard GRBs and long-soft GRBs. The former are supposed to be associated with the merging of compact binaries (Eichler et al., 1989), whereas the latter are expected to occur after the cataclysmic collapse of a massive star (Woosley, 1993).

The popular description for GRB emission is the standard model, also termed the fireball model (Rees \& Mészáros, 1992; Mészáros \& Rees, 1997; Panaitescu, Mészáros, \& Rees, 1998). In this framework, GRBs originate from the dissipation of kinetic energy of an ultra-relativistic outflow (with an initial Lorentz factor of 100-1 000), decelerated by interaction with interstellar matter to produce a fading X-ray and optical afterglow. After the energy decreases to some threshold, the radiation beam is wider than the jetted outflow, so the afterglow becomes observable from angles greater than the high-energy jet.

The fireball model has considerable deficiencies and challenges. For example, the energy budget deduced from the observed emissions and the measured distance implies an onjet-beamed geometry to keep the energy budget physically reasonable (Rhoads, 1999). However, Swift has put this geometry in question (Meszaros, 2006), allowing for exotic theoretical models (Gehrels et al., 2008) that go-beyond standard relativity. Here, only coordinated multi-spectral observations starting a few seconds after the trigger can test theories.

\subsection{Zadko telescope GRB afterglow observations}

The implementation of a robotic telescope for fully automated, calibrated imaging of GRBs is complex. The telescope must respond automatically to alerts sent by satellites through the Gamma-ray burst Co-ordinated (GCN) network (Barthelmy et al., 1994), if all the environmental conditions are fulfilled. These conditions include the time of day (observations are at night), the position of the GRB on the sky, and the priority of the observation. Because of these limits, not all alerts can be followed-up.

Second, there is no manual intervention at the time of the automated triggered imaging to confirm if the sky is clear and the photometry is calibrated. About a third of the sources followed-up are not useful, either because of cloud cover ora technical problem corrupted the photometry. For the Zadko Telescope GRB afterglow follow-up campaign, we list the successfully imaged bursts in Table 2 .

One can clearly see in the distribution of observation times 'holes' corresponding to major refurbishments of the instrument, such as the installation of a new robotic observatory in 2012 (see above). Despite this, and other technical 
Table 2. Zadko telescope follow-up of GRBs. The localizations are obtained from the Swift X-ray Telescope (XRT) position, with an uncertainty of 1.5 arcsec, or the Swift Burst and Transient detector (BAT; denoted by a $\gamma$ ), with an uncertainty of $1^{\circ}-4^{\circ}$. XRT observations were not available for triggers marked with a $\gamma$. All times are given relative to the trigger time, and we report in this table only the upper limits (see Table 3 for the measurements). Zadko employs the same imaging strategy as TAROT: The first image is a $60 \mathrm{~s}$ exposure with tracking off (trailed images), followed by 30, 60, 120, and $180 \mathrm{sec}$ exposures in the tracking mode. For late follow-up (hours post trigger), the $180 \mathrm{sec}$ exposures are stacked. The label 'ld' indicates that the data were lost during a computer update.

\begin{tabular}{|c|c|c|c|c|c|c|}
\hline Burst & $\begin{array}{l}\text { Triggering } \\
\text { satellite }\end{array}$ & $\begin{array}{l}\text { Position } \\
\text { (RA-Dec) }\end{array}$ & $\begin{array}{l}\text { Observation time } \\
\text { [Start-End (s)] }\end{array}$ & $\begin{array}{l}\text { Detection } \\
\text { (Yes/No) }\end{array}$ & $\begin{array}{l}\text { Upper } \\
\text { limit }\end{array}$ & Reference \\
\hline 081118 & Swift & $053022-431804$ & $7056-10800$ & $\mathrm{Y}$ & - & GCNC 8675 \\
\hline 090205 & Swift & $144339-275110$ & 63 360-64 800 & $\mathrm{Y}$ & - & GCNC 8976 \\
\hline 090313 & Swift & $131336+080550$ & $15336-16920$ & $\mathrm{Y}$ & - & GCNC 8996 \\
\hline 090509 & Swift & $160541-282306^{\gamma}$ & $28800-29700$ & $\mathrm{~N}$ & 17.6 & GCNC 9363 \\
\hline 090516 & Swift & $091303-115116$ & $16488-16524$ & $\mathrm{Y}$ & - & GCNC 9380 \\
\hline 090927 & Swift & $225554-705849$ & $7308-7848$ & $\mathrm{Y}$ & - & GCNC 9956 \\
\hline 091127 & Swift & $022620-185708$ & $66600-68040$ & $\mathrm{Y}$ & - & GCNC 10238 \\
\hline 091221 & Swift & $034311+231429$ & $64195-161516$ & $\mathrm{~N}$ & ld & - \\
\hline 100424 & Swift & $135749+013219$ & $153710-268123$ & $\mathrm{~N}$ & ld & - \\
\hline 100628 & Swift & $150346+313911^{\gamma}$ & $7904-277914$ & $\mathrm{~N}$ & 21.0 & - \\
\hline 100702 & Swift & $162246-563256^{\gamma}$ & $32801-302371$ & $\mathrm{~N}$ & ld & - \\
\hline 100704 & Swift & $085434-241210$ & $23571-290112$ & $\mathrm{~N}$ & ld & - \\
\hline $101011 \mathrm{~A}$ & Swift & $031311-655854$ & $3924-4104$ & $\mathrm{~N}$ & 21.5 & GCNC 11336 \\
\hline $101024 \mathrm{~A}$ & Swift & $042602-771555$ & $214.8-222$ & $\mathrm{Y}$ & - & GCNC 11382 \\
\hline $120320 \mathrm{~A}$ & Swift & $161004+084147$ & $24480-26640$ & $\mathrm{~N}$ & 20.7 & GCNC 13087 \\
\hline 120327A & Swift & $162728-292454$ & $149472-151200$ & $\mathrm{Y}$ & - & GCNC 13164 \\
\hline $120422 \mathrm{~A}$ & Swift & $090739+140106$ & $12960-17280$ & $\mathrm{Y}$ & - & GCNC 13250 \\
\hline $121226 \mathrm{~A}$ & Swift & $111434-302423$ & 63-335 & $\mathrm{N}$ & 19.4 & GCNC 14107 \\
\hline $130131 \mathrm{~A}$ & Swift & $112431+480434$ & $71-5400$ & $\mathrm{~N}$ & 16.5 & GCNC 14161 \\
\hline $130206 \mathrm{~A}$ & Swift & $092131-581137$ & $106-482$ & $\mathrm{~N}$ & 20.1 & GCNC 14185 \\
\hline $130313 \mathrm{~A}$ & Swift & $154539-002208$ & $52-320$ & $\mathrm{~N}$ & 19.3 & GCNC 14295 \\
\hline $130315 \mathrm{~A}$ & Swift & $103012-514740$ & $4392-5544$ & $\mathrm{~N}$ & 20.4 & GCNC 14330 \\
\hline $130408 \mathrm{~A}$ & Swift & $085737-322140$ & $50364-52416$ & $\mathrm{~N}$ & 20.5 & GCNC 14372 \\
\hline $130427 \mathrm{~A}$ & Swift & $113233+274152$ & $12420-13140$ & $\mathrm{Y}$ & - & GCNC 14468 \\
\hline $130612 \mathrm{~A}$ & Swift & $171911+164311$ & $33480-36720$ & $\mathrm{~N}$ & 21.2 & GCNC 14885 \\
\hline $130615 \mathrm{~A}$ & Swift & $181919-680940$ & $5760-17280$ & $\mathrm{Y}$ & - & GCNC 14906 \\
\hline $131205 \mathrm{~A}$ & Swift & $084631-600921$ & $13680-16200$ & $\mathrm{~N}$ & 20.2 & GCNC 15579 \\
\hline $131218 \mathrm{~A}$ & INTEGRAL & $073507-644414^{\gamma}$ & $75168-76248$ & $\mathrm{~N}$ & 20.5 & GCNC 15603 \\
\hline 140129A & Swift & $023134-013544$ & $32472-293458$ & $\mathrm{~N}$ & 17.7 & - \\
\hline $140301 \mathrm{~A}$ & Swift & $043814-541524$ & 70-74 880 & $\mathrm{Y}$ & - & GCNC 16064 \\
\hline $140302 \mathrm{~A}$ & Swift & $165526-125242$ & $34655-306638$ & $\mathrm{~N}$ & 17.7 & - \\
\hline $140311 \mathrm{~A}$ & Swift & $135713+003831$ & $120-1111$ & $\mathrm{Y}$ & - & GCNC 15952 \\
\hline $140323 \mathrm{~A}$ & Swift & $234750-795416$ & $3600-5760$ & $\mathrm{~N}$ & 20.8 & GCNC 16059 \\
\hline $140331 \mathrm{~A}$ & Swift & $085928+024302$ & $29736-31248$ & $\mathrm{~N}$ & 20.1 & GCNC 16057 \\
\hline $140628 \mathrm{~A}$ & Swift & $024240-002306$ & $24192-26172$ & $\mathrm{~N}$ & 19.9 & GCNC 16474 \\
\hline 140719B & Swift & $023855-023302^{\gamma}$ & $85-446$ & $\mathrm{~N}$ & 19.0 & GCNC 16610 \\
\hline 141017A & Swift & $061431-583457$ & $2520-2700$ & $\mathrm{~N}$ & 22.4 & GCNC 16920 \\
\hline $141212 \mathrm{~A}$ & Swift & $023630+180849$ & $123-712$ & $\mathrm{~N}$ & 20.3 & GCNC 17184 \\
\hline $150103 \mathrm{~A}$ & Swift & $084640-485309$ & $122-347$ & $\mathrm{~N}$ & 18.9 & GCNC 17273 \\
\hline
\end{tabular}

issues, the Zadko telescope detected $11 \mathrm{GRBs}$ in optical out of 40 successful follow-ups during its $6 \mathrm{yr}$ campaign. This is a typical rate for a robotic instrument (see for instance Klotz et al., 2009, for a comparison with the TAROT instruments).

\subsection{Automated response time, detections, and photometry}

The response time is a key parameter for all robotic telescopes. It will constrain the main scientific drivers in a given field. Comparing the shortest possible response time with the distribution of GRB durations (Kouveliotou et al., 1993), it is clear that the Zadko telescope cannot be used to study the early prompt phase of normal long GRBs, with a mean duration of the order of $20 \mathrm{~s}$, but can image the dynamic early afterglow phase during the transition from prompt to afterglow. We highlight that the response time has improved to a minimum possible response time of $>30 \mathrm{~s}$, during the last $2 \mathrm{yr}$ because of the new observatory (see above).

The $90 \%$ confidence level limiting magnitude reached by the Zadko telescope is consistent with theoretical expectations shown as the thick blue 


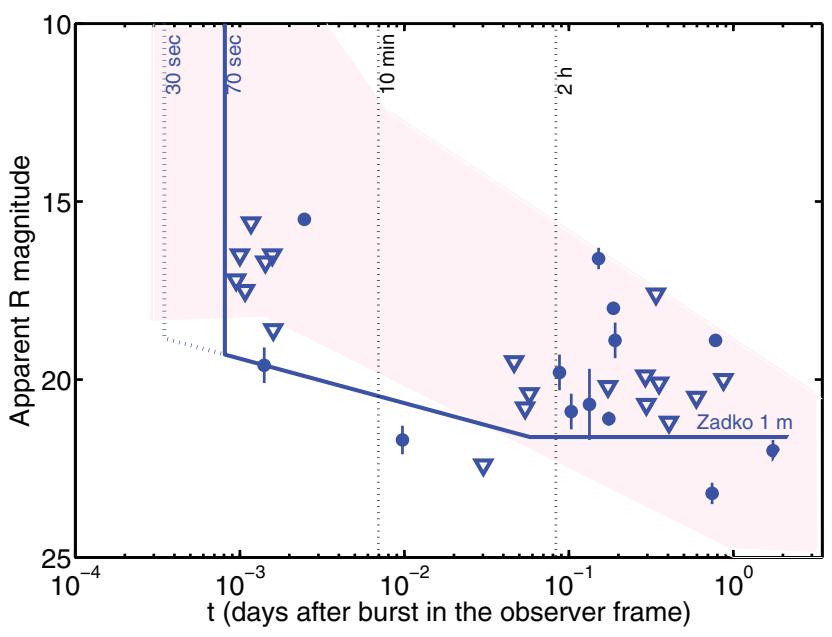

Figure 3. Distribution of GRB afterglow observations and detections by the Zadko telescope. GRB afterglows are represented by filled circles, whereas upper limits are represented as empty triangles. The red shaded area represents the light curves compiled in Kann et al. (2010). The solid blue line is the theoretical observation limits under normal weather conditions with the previous observatory (terminated in 2011). The dashed blue line (at 30 s) is the shortest response time possible for the new observatory (see the electronic version in colour).

line $^{3}$ in Figure 3. It clearly indicates that the telescope cannot probe the entire GRB afterglow flux/luminosity distribution. One day after the burst, only half of the afterglows can be detected. After 3 to $4 \mathrm{~d}$, Zadko cannot detect any afterglows. As a consequence, this constrains the observation strategy against long follow-up delay times ( $1 \mathrm{~d}$ or more) of the afterglow and highlights that the optimal follow-up strategy is a low latency response (comparable to the reaction time). At $1 \mathrm{~d}$ post-alert, it is more practical to search for the host galaxy using deep (stacked) exposures.

In Figure 3, the point representing GRB 090205 is an outlier located below the detection limit. However, it was observed during the commissioning phase with a different camera and settings than the one used after robotization: The detection limit presented in the figure does not apply to this point.

The Zadko telescope can be used to study the so-called dark GRBs (Jakobsson et al., 2004). This subset of GRB afterglows reveals a lack of emission in optical when compared to the X-ray, and their nature still remains puzzling. Because Zadko can obtain upper limits during the first minutes of the burst (up to about 20, see Figure 3), the data can be used to classify a burst as dark (the faintness observed in optical can also be linked to a global faintness observed at all wavelengths), and provide a secondary trigger for a deep follow-up of the GRB location and host galaxy.

Among the sample, several afterglows were imaged, but (for most of them) only for a short duration (typically for

\footnotetext{
${ }^{3}$ This limit is computed for normal weather conditions, but exceptionally good weather can lead to deeper (more sensitive) observations, as shown in the figure.
}

Table 3. GRB afterglows imaged by the Zadko telescope. Filters are labelled ' $C$ ' for clear and ' $\mathrm{R}$ ' for $\mathrm{R}$ band. For clear filter images, the magnitude is expressed in equivalent $\mathrm{R}$ band for comparison with other instruments. The time given relative to the trigger time, and the imaging strategy is the same as described in Table 2.

\begin{tabular}{lccc}
\hline \hline Burst & Time(s) & Filter & Magnitude \\
\hline 081118 & 8856 & $\mathrm{R}$ & $20.9 \pm 0.5$ \\
\hline 090205 & 63360 & $\mathrm{C}$ & $23.2 \pm 0.3$ \\
\hline 090313 & 21346 & $\mathrm{C}$ & $18.02 \pm 0.08$ \\
090313 & 22662 & $\mathrm{C}$ & $18.5 \pm 0.1$ \\
090313 & 24115 & $\mathrm{C}$ & $18.5 \pm 0.1$ \\
090313 & 26028 & $\mathrm{C}$ & $18.62 \pm 0.09$ \\
090313 & 27890 & $\mathrm{C}$ & $18.78 \pm 0.09$ \\
090313 & 29854 & $\mathrm{C}$ & $18.8 \pm 0.2$ \\
\hline 090516 & 16500 & $\mathrm{C}$ & $18.9 \pm 0.5$ \\
090516 & 17101 & $\mathrm{C}$ & $19.5 \pm 0.3$ \\
090516 & 17760 & $\mathrm{C}$ & $20.3 \pm 0.2$ \\
\hline 090927 & 7560 & $\mathrm{R}$ & $19.8 \pm 0.5$ \\
090927 & 9480 & $\mathrm{R}$ & $20.0 \pm 0.5$ \\
\hline 091127 & 67440 & $\mathrm{R}$ & $18.9 \pm 0.1$ \\
\hline $101024 \mathrm{~A}$ & 218.2 & $\mathrm{C}$ & $16.6 \pm 0.3$ \\
$101024 \mathrm{~A}$ & 224.6 & $\mathrm{C}$ & $16.7 \pm 0.3$ \\
$101024 \mathrm{~A}$ & 232.1 & $\mathrm{C}$ & $16.9 \pm 0.3$ \\
$101024 \mathrm{~A}$ & 240.7 & $\mathrm{C}$ & $17.3 \pm 0.3$ \\
$101024 \mathrm{~A}$ & 274 & $\mathrm{C}$ & $17.6 \pm 0.3$ \\
$101024 \mathrm{~A}$ & 320 & $\mathrm{C}$ & $17.8 \pm 0.3$ \\
$101024 \mathrm{~A}$ & 364 & $\mathrm{C}$ & $18.0 \pm 0.3$ \\
$101024 \mathrm{~A}$ & 409 & $\mathrm{C}$ & $18.0 \pm 0.3$ \\
\hline $120327 \mathrm{~A}$ & 150336 & $\mathrm{C}$ & $22.0 \pm 0.3$ \\
\hline $120422 \mathrm{~A}$ & 15120 & $\mathrm{C}$ & $21.1 \pm 0.2$ \\
\hline $130427 \mathrm{~A}$ & 12780 & $\mathrm{R}$ & $15.5 \pm 0.1$ \\
$130427 \mathrm{~A}$ & 13560 & $\mathrm{R}$ & $15.5 \pm 0.1$ \\
$130427 \mathrm{~A}$ & 14640 & $\mathrm{R}$ & $15.4 \pm 0.1$ \\
$130427 \mathrm{~A}$ & 15480 & $\mathrm{R}$ & $15.6 \pm 0.1$ \\
$130427 \mathrm{~A}$ & 16260 & $\mathrm{R}$ & $15.5 \pm 0.1$ \\
$130427 \mathrm{~A}$ & 18690 & $\mathrm{R}$ & $15.6 \pm 0.1$ \\
$130427 \mathrm{~A}$ & 26910 & $\mathrm{R}$ & $16.3 \pm 0.2$ \\
\hline $130615 \mathrm{~A}$ & 11520 & $\mathrm{R}$ & $20.7 \pm 1.0$ \\
\hline $140301 \mathrm{~A}$ & 841 & $\mathrm{C}$ & $21.7 \pm 0.4$ \\
\hline $140311 \mathrm{~A}$ & 223 & $\mathrm{R}$ & $19.6 \pm 0.5$ \\
$140311 \mathrm{~A}$ & 282 & $\mathrm{R}$ & $19.9 \pm 0.3$ \\
$140311 \mathrm{~A}$ & 427 & $\mathrm{R}$ & \\
$140311 \mathrm{~A}$ & 821 & & \\
\hline \hline & & & \\
\hline
\end{tabular}

about 1 to $2 \mathrm{~h}$ ). In addition, most sources were visible with a delay of several hours. Only four events were followed-up a few tens of seconds after the trigger and lasting for more than 3 h: GRB 090313, GRB 101024A, GRB 130427A, and GRB 140311A. We list all the observations and detections in Tables 2 and 3, respectively. It is beyond the scope of this paper to present a detailed analysis of each burst observed by the Zadko telescope, but more detailed studies have been undertaken (e.g. Gendre et al., 2011,for GRB 101024A).

\subsection{Further GRB science opportunities}

The response time and limiting magnitude set the potential for breakthrough science in relation to testing the standard 
fireball model. Potential new science and discoveries from Zadko telescope automated triggered imaging include:

- The spectral evolution of the prompt phase. The Zadko telescope will not compete with faster robotic instruments for observing the start of the prompt phase, as can be noted in Figure 3; however, its response time and sensitivity could probe the origin of the early optical emissions prompt phase of the brightest and longest duration GRBs.

- The plateau phase. One of the main results from the Swift satellite is the presence of a plateau phase seen in X-ray between the prompt and the afterglow phases. The nature of this plateau is debated, and the comparison with optical data is critical to test the current models. Boër, Gendre, \& Stratta (2015) have shown that the distribution of the end of the prompt phase in X-ray peaks at about $100 \mathrm{~s}$. Accordingly, Willingale et al. (2007) have shown that this plateau phase ends about $10^{4} \mathrm{~s}$ after the trigger. Thus, there is a significant fraction of bursts with the plateau phase starting within the window of Zadko telescope triggered imaging times.

- Population III stars. The first stars are metal-poor and proposed relics of the early Universe. They should form at very large redshift ( $z \sim 10-20$, Bromm \& Loeb, 2006), but could rarely be present at $z \sim 2-4$ (Tornatore, Ferrara, \& Schneider, 2007), and should produce GRBs (Suwa \& Ioka, 2011; Nakauchi et al., 2012). Moreover, isolated blobs of original matter, without metals, could be present near the Milky Way, and could form, if disturbed, new population III stars (Morales-Luis et al., 2011). The properties of these GRBs are expected to be similar to ultra-long GRBs (Gendre et al., 2013; MacPherson, Coward, \& Zadnik, 2013), with a prompt phase lasting more than $600 \mathrm{~s}$ (indicated by the $10 \mathrm{~min}$ line in Figure 3).

\section{SOLAR SYSTEM SCIENCE - ASTEROID PHOTOMETRY}

Measuring brightness variations is the primary source of information on an asteroid's shape. Photometric light curves also provide other very useful physical parameters, such as the rotation period and the pole coordinates. Determination of these physical properties requires photometry at different geometries, i.e. over several oppositions (Devogèle et al., 2015).

Combining these observations with other measurements of the size (for instance by thermal modelling or stellar occultation), a more accurate volume, and density, can be determined (Carry, 2012). Also, shape is an indicator for the past collisional history of an asteroid. Without knowledge of an asteroid's shape, application of thermo-physical models to derive thermal inertia and size is affected by significant uncertainties.
Currently, only the brightest asteroids have been systemically observed during several oppositions. This is mainly the result of the use of a large number of small telescopes i.e. 20$40 \mathrm{~cm}$ (Mousis et al., 2014). Photometry is also obtained as a by-product of other observations, such as asteroid astrometry, or large sky surveys. In such cases, however, complete light curves are generally not obtained, but only sparse photometry. Although it has been shown that sparse data can be exploited for the goals mentioned above, the quality of the data requires a careful selection and the process does not work for all the objects (Hanus et al. 2013). As of late 2015, $\sim 400$ detailed asteroid shapes ${ }^{4}$ (see Durech, Sidorin, $\&$ Kaasalainen, 2010,using $\sim 7 \times 10^{5}$ known asteroids) and $\sim 2000$ rotational period measured, with rough shape parameters (Harris, Warner, \& Pravec, 2012).

Unfortunately, very few medium size telescopes (meterclass) are systematically exploited for asteroid photometry. In comparison to the largest telescopes ( $4 \mathrm{~m}$ and more), they can offer more flexibility of use and are generally available for a longer time. Also, their sensitivity considerably extends the number of asteroids available for photometry. The Zadko telescope is ideal for performing sensitive photometry using the robotic scheduler.

Another important issue for asteroid observations is the measurement of accurate position in the sky. Several asteroids are usually in the FoV of the Zadko Telescope, when pointing near the ecliptic position. Reporting of the astrometric measurements of such asteroids will lead to an improvement of the asteroid orbit database. Furthermore, Zadko is a part of a network of facilities that will follow-up Gaia (ESA satellite for astrometry and photometry) asteroid alerts in the coming years (Todd et al., 2013). In parallel with this work, new models that constrain optimal search areas and imaging cadences for narrow and wide-field survey telescopes was investigated (Todd et al., 2012a, 2012b). We detail below recent programs in active development employing the Zadko telescope for asteroid photometry.

\subsection{L-type and Barbarian asteroids}

Asteroids belonging to the $\mathrm{L} / \mathrm{K}$ taxonomic classes could hold the key to understand the early phases of formation of the Solar System, when the solid phase in the proto-planetary nebula, which was largely dominated by highly refractory material, which solidify at the highest temperatures. Recent numerical simulations (Johansen and Klahr, 2011) suggest that, in those conditions, typical of the first $\sim 1 \mathrm{Myr}$ after the disk formation, local concentrations of solid grains could have been rapidly assembled in large planetesimals of $\sim 100$ $\mathrm{km}$. Recently, near-IR observations (Sunshine et al., 2008) have suggested that some asteroids could also include in their composition a high fraction of that primordial material, hence be extremely ancient. A large network led by P. Tanga recently

\footnotetext{
${ }^{4}$ see e.g. the DAMIT data base:http://astro.troja.mff.cuni.cz/projects/ asteroids3D/web.php
} 
started an extensive activity of physical characterization of those asteroids, the so-called Barbarians, from the name of (234) Barbara, the first of this type discovered (Cellino et al., 2006). The anomaly leading to the identification of the Barbarians was the peculiar variation of their linear polarization with the phase angle (the angle between the directions to the Sun and to the Observer, as seen from the asteroid).

A total of 13 Barbarians have been found so far (Gil-Hutton et al., 2008; Masiero \& Cellino, 2009; Cellino et al., 2014). Seven of them are in the Watsonia dynamical family, caused from the disruption of a single parent body (Cellino et al., 2014). In terms of taxonomy, based on their similar spectrophotometric properties, Barbarians are classified as members of the unusual classes: $L, L d$, and $K$.

In absence of solid theoretical models capable of explaining the observed polarization, Cellino et al. (2006) suggested, as a speculative explanation, that anomalous polarization could also be due to large-scale concavities responsible for causing an anomalous distribution of incident sunlight and scattering angles. In this respect, (234) Barbara has a very irregular shape and reveals large concavities, as shown by Delbo et al. (2009), and later confirmed by a more extensive analysis using different techniques (Tanga et al., 2015).

For the small sample of known rotation periods of the largest Barbarians (seven objects), all of them are slow rotators (period 12-24 $\mathrm{h}$ or more) with respect to the average period of similar-sized asteroids (5-6 h). Slow rotation periods and the possible presence of concavities are indicators of past collisional history. However, the verification of this hypothesis requires a large amount of data on rotations and shapes. One tool for determining rotation periods and indications of shape irregularities is to obtain light curves spanning a near complete period.

\subsubsection{Zadko photometry of asteroids}

As the rotation period of the so-called slow rotators generally exceeds the duration of night on Earth, telescopes distributed across many longitudes are required. Ideally, a light curve should be obtained over $\sim 1$ week; otherwise the changing illumination/observation geometry will make its interpretation more complex. For these reasons, Zadko is exceptionally well placed relative to other instruments that have also been employed (in France, Poland, USA, and Chile). We note that this type of observation requires blocks of dedicated time, several hours of imaging spread over a night. To achieve this task automatically on the Zadko telescope, we created a high priority schedule that could only be interrupted by a GRB alert.

During $1 \mathrm{yr}$ (2014 to 2015), the Zadko telescope contributed to the period determination for 11 asteroids. These objects (and most of the known asteroids) do not have measured rotation periods, or at best preliminary ones with unconfirmed values (with accuracies of the order of $0.1 \mathrm{~d}$ or worse) obtained from partial light curves not covering full rotations. Figure 4 illustrates a typical example, in which Zadko observations covers a portion of a light curve observable only from

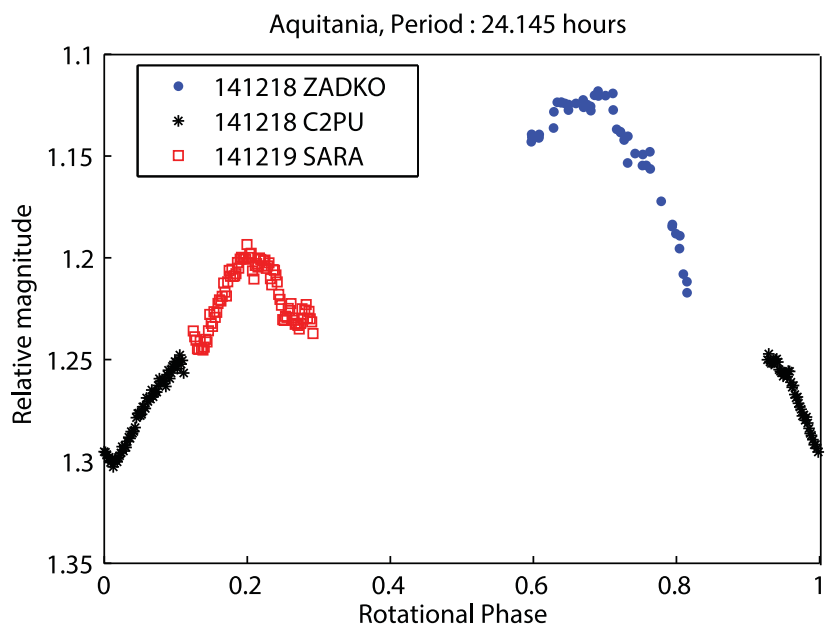

Figure 4. Light curve of (387) Aquitania. The observations by the Zadko telescope provided light curve measurements around the brightness peak, unobserved at other longitudes. With a rotation period close to $24 \mathrm{~h}$, a single site can only acquire partial light curves.

Australia, thus capturing the maximum of brightness during a single rotation. The European longitudes of $\mathrm{C} 2 \mathrm{PU}$ close to Nice, France ensured the coverage of the minimum over the same rotation of the asteroid.

By obtaining the near complete light curves, we were able to measure rotation periods with uncertainties better than $1 \times 10^{-3}-1 \times 10^{-4} \mathrm{~d}$ with a measured dispersion $<0.01 \mathrm{mag}$ nitudes for targets of relatively high brightness $(\mathrm{V} \sim 11-13)$. This accuracy exceeds the photometric accuracy required for deriving most asteroid rotation periods (having typical amplitude variation of $0.2-1 \mathrm{mag}$ ). Zadko photometry contributed to the shape determination of (234) Barbara (Tanga et al., 2015), and updated results are due to appear in a paper in preparation.

\section{ANTARES FOLLOW-UP}

ANTARES is the largest high-energy neutrino telescope in the Northern Hemisphere (Ageron et al., 2011) and is aimed at the search for a muon neutrino signal from astrophysical sources. Such a discovery would unambiguously probe the identified source as an efficient cosmic accelerator of hadrons that is a key to solve the mystery of the ultra-highenergy cosmic-ray origin in the universe. A multi-wavelength follow-up of the neutrino alerts has been developed by the ANTARES collaboration (Ageron et al., 2012), in order to quickly detect the EM counterpart from a potential neutrino source from radio to gamma-rays. This program denoted as Telescopes-ANTARES Target of Opportunity (TAToO) provides, in real-time using a socket protocol similar to the GCN system, the coordinates of the most promising neutrino events detected by the ANTARES telescope.

In the optical domain, the Zadko telescope is well matched to the TAToO program for several reasons. 
Table 4. Zadko follow-up of the ANTARES alert in the period 2014-2015. The reaction time corresponds to the time between the moment when the neutrino is detected by the ANTARES detector and the time when the first image is taken by the Zadko telescope. In the case of short time delays the limiting magnitude of the image was calculated. (*) Reaction time is biased $(\sim 1 \mathrm{~d}$ offset) because of an error in the scheduler program.

\begin{tabular}{lcc}
\hline \hline Alert & Reaction time & $\begin{array}{c}\text { New detection } \\
\text { Yes/No }\end{array}$ \\
\hline ANT140123A & $2.12808 \mathrm{~d}$ & $\mathrm{~N}$ \\
ANT140125A & $1.36891 \mathrm{~d}$ & $\mathrm{~N}$ \\
ANT140126A & $0.96329 \mathrm{~d}$ & $\mathrm{~N}$ \\
ANT140202A & $1.25782 \mathrm{~d}$ & $\mathrm{~N}$ \\
ANT140227A & $6.65249 \mathrm{~d}$ & $\mathrm{~N}$ \\
ANT140228A & $5.80076 \mathrm{~d}$ & $\mathrm{~N}$ \\
ANT140301A & $\mathbf{6 4 . 4 1 2} \mathrm{s}$ & $\mathrm{N}(\mathrm{R} \geq 19.8)$ \\
ANT140304A & $1.32959 \mathrm{~d}$ & $\mathrm{~N}$ \\
ANT140309A & $1.45869 \mathrm{~d}$ & $\mathrm{~N}$ \\
ANT140311A & $2.30843 \mathrm{~d}$ & $\mathrm{~N}$ \\
ANT140323A & $\mathbf{1 5 4 . 9 7 6} \mathrm{s}$ & $\mathrm{N}(-)$ \\
ANT140505A & $59.96 \mathrm{~d}$ & $\mathrm{~N}$ \\
ANT140619A & $1.11634 \mathrm{~d}$ & $\mathrm{~N}$ \\
ANT140630A & $2.33353 \mathrm{~d}$ & $\mathrm{~N}$ \\
ANT140818A & $45.3101 \mathrm{~d}$ & $\mathrm{~N}$ \\
ANT140914A & $5.29319 \mathrm{~d}$ & $\mathrm{~N}$ \\
ANT140925A & $5.20087 \mathrm{~d}$ & $\mathrm{~N}$ \\
ANT141027A & $6.16642 \mathrm{~d}$ & $\mathrm{~N}$ \\
ANT141112A & $6.82595 \mathrm{~d}$ & $\mathrm{~N}$ \\
ANT141220A & $\mathbf{5 2 . 8 9 6 \mathbf { s }}$ & $\mathrm{N}(\mathrm{R} \geq 20.0)$ \\
ANT150111A & $4.31658 \mathrm{~d}$ & $\mathrm{~N}$ \\
ANT150122A & $6.49385 \mathrm{~d}$ & $\mathrm{~N}$ \\
ANT150131A & $9.23907 \mathrm{~d}$ & $\mathrm{~N}$ \\
ANT150213A & $4.26905 \mathrm{~d}$ & $\mathrm{~N}$ \\
ANT150224A & $1.43219 \mathrm{~d}$ & $\mathrm{~N}$ \\
ANT150321A & $15.7736 \mathrm{~d}$ & $\mathrm{~N}$ \\
ANT150324A & $10.5152 \mathrm{~d}$ & $\mathrm{~N}$ \\
\hline \hline & & \\
\hline \hline
\end{tabular}

1. It is located where the maximum fraction of ANTARES alerts are visible, see Figure 5.

2. Because of its rapid response to the alert system, it can quickly observe the neutrino alert position in the sky. This feature is very important if the neutrino signal is produced by a rapidly fading transient source such as a GRB.

3. The limiting magnitude of the Zadko telescope $(\sim 21$ in $60 \mathrm{~s}$ of exposure in a clear night without a bright moon) can place stringent limits on the optical brightness of the potential neutrino source.

From 2013, the Zadko telescope has participated in the robotic telescope network of the TAToO program, and succeeded in responding to 58 neutrino alerts. Among these, three were rapidly followed-up in less than $160 \mathrm{~s}$ after the neutrino trigger (ANT140301A : $64 \mathrm{~s}$, ANT140323A : $155 \mathrm{~s}$ and ANT141220A : 53 s) see Table 4. Unfortunately, no new transient sources were observed, but upper limits in $\mathrm{R}$ magnitude were derived for ANT140301A $(\mathrm{C} \geq 19.8)$ and
ANT141220A ( $C \geq 20)$. No reliable limiting magnitude could be obtained for ANT140323A images because of their poor quality. In the framework of GRBs, the Zadko telescope is able to exclude a typical bright GRB origin for the two neutrinos ANT140301A and ANT141220A, because most GRB afterglows are typically brighter at $60 \mathrm{~s}$ than the limits we measure, see Table 4. However, the GRB origin cannot be completely excluded because the GRB progenitor could also be intrinsically sub-luminous, or embedded in a dense circum-stellar medium. For the 55 late follow-up alerts ( $\geq 1 \mathrm{~d}$ after the neutrino trigger), no new transient source was discovered and no strong constraints on the GRB origin could be set because the major part of the GRB afterglow population is below the limiting magnitude of the Zadko Telescope $1 \mathrm{~d}$ after the $\gamma$-ray prompt emission.

As shown in Table 4, the response time of the Zadko Telescope can exceed $2 \mathrm{~d}$. This long response time is due to observational constraints (the field of view is not immediately observable due to its position in the sky, the field of view is too close to the moon) or bad weather conditions (clouds, rain, wind). However, a problem in the scheduler program was detected and prevents us from following-up 7/55 alerts with shorter reaction time. Because these alerts were received during the day, the first observation was reported to the next night, i.e. one day after the receipt of the ANTARES alert. Thus, the reaction time of the Zadko telescope was biased and no alert could be followed-up in a delay of few minutes to few hours. This problem was solved in February 2015 with an update of the observational strategy of the ANTARES alert follow-up. The new program is now fully optimised (in response time) to detect a potential optical counterpart.

\section{SUPERNOVAE SEARCH}

Supernovae $(\mathrm{SNe})$ are energetic transients related to either binary systems, implying an accreting white dwarf (Type Ia, see Whelan and Icko, 1973; Nomoto, 1982; Maoz, Mannucci, \& Nelemans, 2014,for a recent review) or the collapse of massive stars (Type II-Ib-Ic, see Janka et al., 2012). Type Ia $\mathrm{SNe}$ are widely used as cosmological probes (Perlmutter et al., 1999; Riess et al., 1998) as they can represent standard candles. Core collapse supernovae (CCSNe) still raise many unanswered questions concerning their origin, including: What are the properties of the stellar progenitors, and those of the newly born compact stellar remnants? What is the efficiency of the nucleosynthesis in massive stellar explosions? What would be the GW signal from a stellar collapse? Since the discovery of the first neutrino signal from the core collapse SN1987A, and more recently the discovery of the Xray/UV signature of the shock breakout in SN2008D (Soderberg et al., 2008) type II supernovae are an active research area.

Because $\mathrm{SNe}$ are relatively rare events in the Universe (about $1 \mathrm{SN} / \mathrm{gal} / 100 \mathrm{yr}$ ), the typical strategy to detect them consists of observing a large number of galaxies each night which implies scanning a large part of the sky each night. 


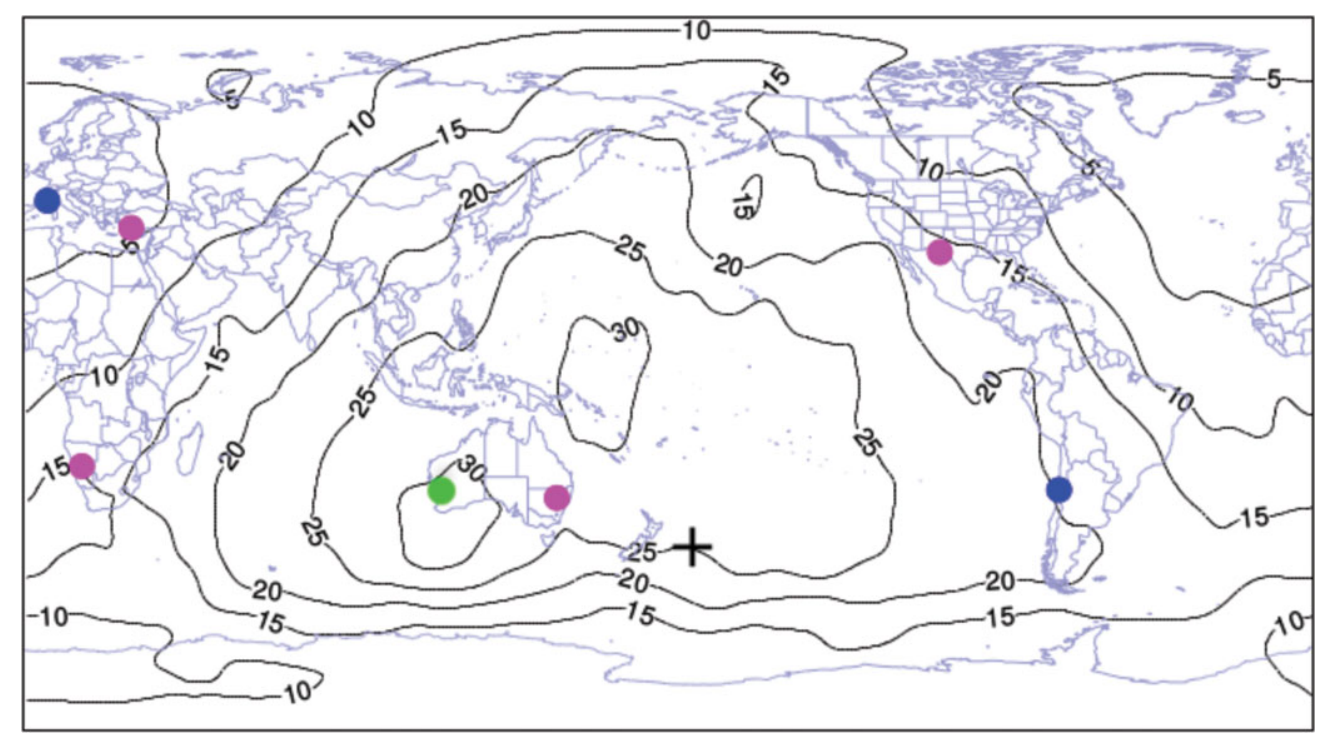

Figure 5. The contours on the world map indicate the percentage of neutrino triggers visible at low latency. (Based on 140 ANTARES alerts). The black cross indicates the antipodal point for the ANTARES experiment. The TAToO robotic follow-up network is shown during the period 2012-2014 : (blue) ROTSE telescopes, (magenta) TAROT, and Zadko telescopes (green).

Because of this strategy, and the growing number of facilities, about 200-300 SNe have been discovered each year for the last 15 yr. However, because these discoveries often occur when the supernova is already in its declining phase, the features of the early rising supernovae are missing.

As shown in Soderberg et al. (2008), in the case of CC$\mathrm{SNe}$, detailed observations of the early phase of the supernovae (few hours to few days after the explosion) will help to understand the explosion mechanism, and to put constraints on the photospheric radius of the stellar progenitor, which is one of the key parameters to understand the phenomenon. It would also help to improve numerical simulations of CC$\mathrm{SNe}$, as there is sparse data covering this early phase. Finally, observing the early stage of the SN explosion is important to estimate the total luminosity, the age of the $\mathrm{SN}$, and the early time evolution of the SN spectrum.

\subsection{Zadko supernova follow-up}

Because the field of view of the Zadko Telescope cannot scan a large part of the sky, an innovative SN search strategy has been developed. The goal is to detect $\mathrm{SNe}$ during the early stage of the explosion, and to follow-up until they reach their maximum brightness $(\sim 20 \mathrm{~d}$ after the explosion) with a high sampling. For some fraction of CCSNe, we expect to detect the tail of the thermal shock breakout emission in the optical/near-UV domain $(\sim 1 \mathrm{~d}$ after the explosion) as already observed for a few of CCSNe, SN1987A (Hamuy \& Suntzeff, 1990), SN1993J (Richmond et al., 1994), SN1998bw (Galama et al., 1998), SN1999ex (Stritzinger et al., 2002), and SN2008D (Modjaz et al., 2009). To achieve this, we designed a catalogue of nearby galaxies of interest which historically are prolific in producing $\mathrm{SNe}$, or for which detection $\mathrm{SN}$ is easier, i.e. face-on galaxies. After studying the properties of 1000 galaxies that have produced $\mathrm{SNe}$ since $1885^{5}$, we conclude that the most prolific galaxies include:

1. Nearby galaxies $(\leq 40 \mathrm{Mpc})$ with large angular radius ( $\geq 4$ arcmin)

2. Face-on galaxies

3. Starburst or HII galaxies that have an enhanced stellar formation

4. Spiral galaxies or merging galaxies

According to the above criteria, we would expect to detect 7-10 SNe per year from our catalogue of 112 galaxies. However, taking into account the variable weather conditions, the moon phases and expected technical difficulties, we should observe 2-3 SN during their early phase with good observational conditions. In order to optimise the number of observed galaxies per night, we specify an exposure time for each galaxy to detect a SN to a limiting absolute magnitude of $R_{\mathrm{abs}} \sim-13$. This limiting magnitude is deep enough to detect faint sources like young $\mathrm{SNe}$ without consuming too much observation time.

The program was implemented in June 2014 and in 2014-2015, $8 \mathrm{SNe}$ were discovered in galaxies belonging to our catalogue: SN2014A (NGC5054), SN2014B (NGC4939), SN2014C (NGC7331), SN2014J (NGC3034), SN2014L (NGC4254), SN2014df (NGC1448), ASASSN2014ha (NGC1566), and SN2014dt (NGC4303). Among them, six were classified as $\mathrm{CCSNe}$ and two as Type

\footnotetext{
5 see http://www.cbat.eps.harvard.edu/lists/Supernovae.html
} 

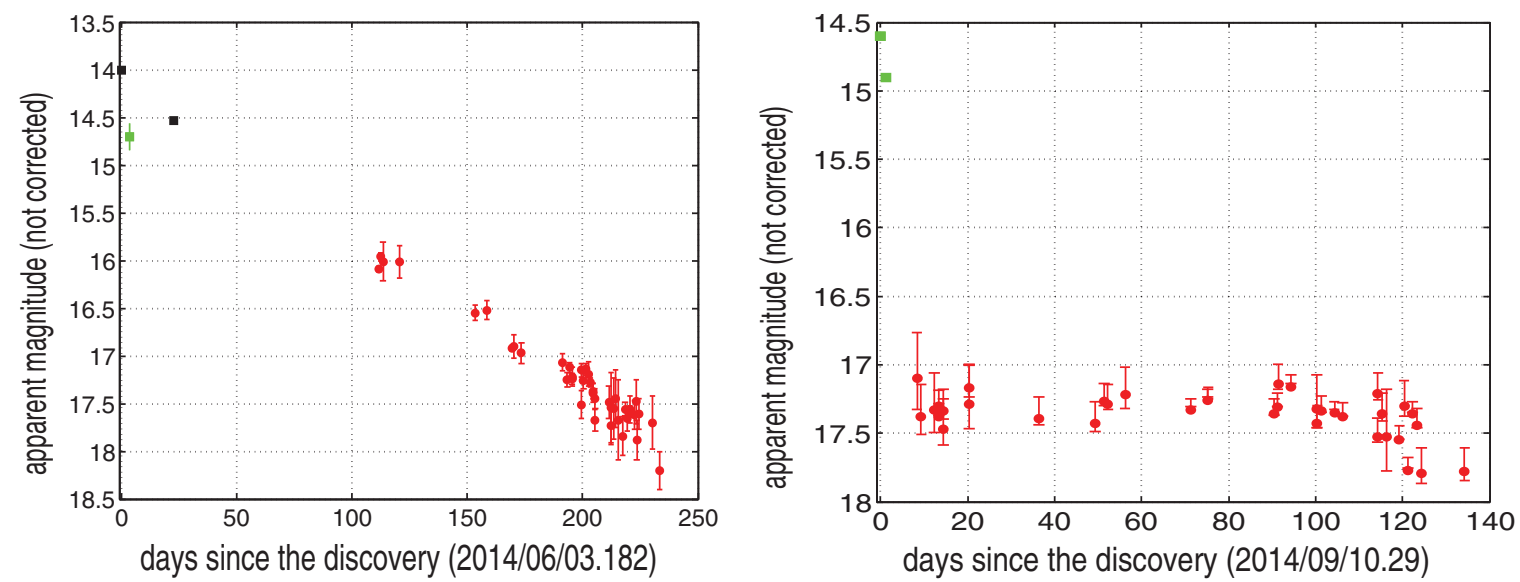

Figure 6. Light curves of SN2014df (left) and SN2014ha (right) with R-band (red), V-band (green), and unfiltered observations (black). The Zadko photometry, not corrected for Galactic dust extinction and converted to R and V filters, are shown as filled circles. Discovery of SN2014df and SN2014ha (squares), were made by Berto Monard ( $\pm 0.14 \mathrm{mag}$ ), and the All Sky Automated Survey for SuperNovae $(V \approx 14.6$ and 14.9 , no errors provided), respectively.

Ia SNe. Unfortunately, the first five $\mathrm{SN}$ were discovered 4 months before the program commenced. Due to observational constraints, the Zadko Telescope detected the three other supernovae (SN2014df, ASAS-SN2014ha and SN2014dt) days after their discovery (see Figure 6 for light curves of SN2014df and SN2014ha). Example images can be found at http://www.rochesterastronomy.org/sn2014, http:// www.astronomerstelegram.org/?read=6460 and http://www. rochesterastronomy.org/sn2014/.

Since the beginning of the SN program, the number of SN discovered per galaxy is in good agreement with the expected rate. So far, the project has not detected an early SN, which we attribute to a poor duty cycle from weather conditions, technical upgrades and observational constraints. The project will continue to search for young and nearby $\mathrm{SNe}$ with a future update of the targeted galaxy catalogue.

\section{OPTICAL FOLLOW-UP OF GRAVITATIONAL WAVE TRIGGERS}

The first detections of GWs from coalescing black hole binary systems: GW150914 (Abbott et al., 2016c) and GW151226 (Abbott et al., 2016a), in late 2015 opened up a new observational window to the Universe. As GWs propagate virtually freely through space, they can provide a pathway to dynamic astrophysical processes presently inaccessible by EM detection. Among the strongest emitters in the $\mathrm{GW}$ spectrum are cataclysmic sources, such as coalescing systems of compact binaries and the collapse of massive stars. Future detections may have associated emissions in the EM spectrum allowing the opportunity for coordinated multi-wavelength observations.

The GW detector network is being led by Advanced LIGO (aLIGO; Abadie et al., 2010) and will be followed by Advanced Virgo (AdV Acernese et al., 2015) in 2017. When these advanced detectors reach design sensitivity (around
2019), they will have 10 times greater sensitivity than the first generation instruments (2003-2009) yielding a factor 1000 improvement in observed volume. These facilities can detect coalescing systems of neutron stars to distances approaching $\sim 400 \mathrm{Mpc}$, neutron star-black hole systems to $\sim 1 \mathrm{Gpc}$ and stellar mass black hole coalescences like GW150914 out to cosmological distances greater that $z \sim 1$.

The coordinated follow-up of GW events in the EM spectra and beyond will increase the confidence of detections, maximise the scientific output, and allow more sensitive searches into GW data, extending the sensitivity horizon. As the detector network sensitivity improves incrementally in stages towards design sensitivity, we expect to probe other source populations, such as binary neutron star and neutron starblack hole mergers. Such joint detections will initiate a new era of multi-messenger astronomy.

\subsection{The aLIGO/AdV follow-up program}

For the first aLIGO observation run (O1 that ran from September 2015 to January 2016), Zadko was one of a number of EM facilities selected for follow-up GW sources. The LIGO/Virgo collaboration had over 63 worldwide EM partners with signed MoUs for conducting follow-ups of GW triggers at that time; around $70 \mathrm{MoUs}$ will be in place for the second observation run in late 2016 (O2). Of the partners, $73 \%$ are in the UV/Optical/IR, $11 \%$ in the radio and the remainder are in high energy X-ray and Gamma-ray. Australian involvement includes MWA (Tingay et al., 2013); ASKAP (VAST) (Murphy et al., 2013); AAT (Tinney et al., 2004); SkyMapper (Keller et al., 2007), GOTO ${ }^{6}$ and the 'Deeper Wider Faster' project that targets simultaneous, fast cadenced observations through shadowing of optical and radio fields; interesting candidates are followed up using a number of

\footnotetext{
${ }^{6}$ http://goto-observatory.org/
} 
partner EM facilities, including Zadko, covering low to high energy (Howell et al., 2015). In the high energy gamma-ray, there will be Australian involvement through the Cherenkov Telescope Array (CTA; Acharya et al., 2013), The High Energy Stereoscopic System (H.E.S.S; Lennarz et al., 2013), as well as coordinated neutrino observations using IceCube (IceCube Collaboration et al., 2006).

The main latency bottleneck during $\mathrm{O} 1$ was the automatic and manual verifications that included checks on data quality and conditions. Therefore, to allow alerts to be sent out significantly faster, automation is a particular aim for future science runs in the advanced GW detector era. The statistical significance of a GW trigger is given through its false alarm rate (FAR), which is the rate that false positives appear above a given signal-to-noise ratio threshold. Typical FARs for triggers sent out during the $\mathrm{O} 1$ were of order $1 /$ month.

To rapidly communicate the information on $\mathrm{GW}$ events, the VOEvent $^{7}$ standard was adopted (Williams et al., 2012). The content of a VOEvent alert sent out by aLIGO for a compact binary coalescence event included estimates of the FAR (in $\mathrm{Hz}$ ) and the sky position of the source provided by a way of a probability sky map (which can be multi-modal and nonGaussian). Future runs could include additional information such as estimates of the luminosity distance of the source and an indication of the component masses of the system.

\subsection{The first EM follow-up of a GW transient}

The first confirmed GW transient, GW150914 (a binary black hole merger) was discovered by the aLIGO detectors on 2015 September 14 (Abbott et al., 2016c). Preliminary estimates of the time, significance, and sky location of the event candidate were shared with 63 teams of observers covering radio, optical, near-infrared, X-ray, and gamma-ray wavelengths with ground- and space-based facilities. Following detection through the low-latency burst detection pipelines a Coherent WaveBurst (cWB) skymap, based on minimal assumptions, was available within $17 \mathrm{~min}$; this was followed by more comprehensive LALInference Burst (LIB) skymap within $14 \mathrm{~h}$. Due to additional data integrity and verification checks, these two maps were not circulated till $2 \mathrm{~d}$ post-detection. Of the 63 teams, 25 follow-ups were reported via a private Gamma-ray Coordinates Network Circulars. Details of the skymap production and the follow-up observations (by 25 teams) for this first campaign are outlined in Abbott et al. (2016b); this included a disputed claim of a possible short GRB coincident (within 60 ms of GW150914) with the GW event (Connaughton et al., 2016). Despite over 60 participating facilities with MoUs for EM follow-up, around a third managed to acquire data for this first event. This can be attributed to many factors, including weather (optical), instrumental problems, and rapid follow-up not possible because of longitude separation. For the first follow-up campaign, the only Australian facilities or collaborations to obtain follow-

\footnotetext{
${ }^{7}$ http://www.ivoa.net/documents/VOEvent/
}

up data were SkyMapper and TZAC (TAROT-Chile), of which Zadko (the Australian node) was not operational at the time of the trigger.

\subsection{Gravitational wave follow-up challenges and strategies}

Looking ahead to the second aLIGO/Virgo observation run (02; late 2016), the most likely GW sources to yield optical counterparts are the mergers of coalescing binary systems of neutron star. As discussed in Section 4.1, these events have been strongly suggested as the progenitors of short hard $\gamma$-ray bursts (Tanvir et al., 2013; Berger, Fong, \& Chornock, 2013). The evidence stems from the fact that short-hard GRBs are often observed in older stellar populations with offsets of tens of $\mathrm{kpc}$ from their galactic centres; this is consistent with binary compact object formation channels. Additionally, follow-up observations in the infrared of GRB 130603B provided evidence of a 'kilonova'; a faint transient predicted to form after the merger of two neutron stars, and powered by the radioactive decay of the ejected neutron rich matter (Li \& Paczyński, 1998; Rosswog, 2005; Metzger et al., 2010).

As highlighted during $\mathrm{O} 1$, a particular challenge for optical follow-ups of $\mathrm{GW}$ sources during $\mathrm{O} 2$ and beyond will be error boxes of $100 \mathrm{~s} \mathrm{deg}^{2}$; compare this with the typical error boxes for GRB follow-ups of order arc mins. As mentioned earlier, these error regions are provided to optical facilities as probability sky maps. The morphology of the skymaps is dependent on the location of the source in the sky relative to the antenna pattern function of the GW detector network. The shapes of the probability map can take the form of a single elongated arc that could cover several hundred square degrees, or two or more degenerate arcs that could include small isolated regions. When Virgo increases the network to three-detectors (in 2017) localisations should improve; one can expect confidence regions of $10 \mathrm{~s}$ of degrees in less than $17 \%$ of events (Singer et al., 2014).

To make the most of opportunities, instrument dependent strategies must be employed to cover the most probable regions of the error region in sufficient time to capture a fading EM source. These strategies will have to be adapted depending on the particular morphology and for partially accessible error regions, the component available to the southern hemisphere. The most likely EM counterparts, short hard GRBs have fainter afterglows than the longer duration bursts. However, for on-axis sources within the aLIGO/AdV horizon one would expect these events to be bright enough for identification; however, event rates and beaming considerations suggest that in most cases the bursts would be off-axis requiring deep searches.

Different follow-up techniques to screen candidate counterparts are still in the testing stage; these include simple tiling strategies employing galaxy catalogues to more sophisticated clustering algorithms based on machine learning. Source confusion will also be an important consideration. Sources such as Solar System objects can be excluded by employing a 
cadence of order $10 \mathrm{~s}$ to mins between images of the same field. Supernovae can be excluded by their relatively long durations and flare stars can be monitored for repeated variability. The most important strategy for identifying the type of candidate transients will be coordinating deep follow-up with other facilities (both radio and optical). We are currently in discussions with multiple collaborations with access to globally distributed meter class facilities for the above.

Initial follow-ups by Zadko will employ tiling the highest probability sky regions and secondly target only the brightest galaxies utilizing the current automated catalogue matching pipeline that creates a list of unknown candidates for an alert image $^{8}$. Image subtraction can also be used for galaxies that we have reference images, i.e. from our current supernovae search.

\subsection{Coordinated GW follow-up imaging with Zadko}

With the intense interest in GW follow-up, a number of facilities are being constructed as dedicated instruments with FoVs large enough to cover the GW error regions in reasonable times. One such facility that could be operational during the later stages of aLIGO/AdV is RAMSES; if successful a similar instrument could operate alongside Zadko. The RAMSES prototype will consist of a set of $16 \times 40 \mathrm{~cm}$ telescopes equipped with rapid cameras capable of readout times from $100 \mathrm{~ms}$ to several minutes; it will be able to reach magnitudes of 15 in $1 \mathrm{~s}$ and mag 20 within around $10 \mathrm{~min}$. Each telescope has a $2.5 \mathrm{deg}^{2} \mathrm{FoV}$, resulting in a combined $100 \mathrm{deg}^{2} \mathrm{FoV}$; this in combination with a slew time of around $5 \mathrm{~s}$ would enable instantaneous coverage of a large proportion of a typical aLIGO/AdV error region. RAMSES has received funding and will be based in National Aures Observatory in Algeria ${ }^{9}$ with a prototype built in France.

Comparable instruments employed in GW follow-ups include BlackGEM (40 deg ${ }^{2} \mathrm{FoV}$; mag 22 in 5 min; Bellm, 2014) and the Zwicky Transient Facility (ZTF; $47 \mathrm{deg}^{2}$ FoV; mag 20.5-21 in $30 \mathrm{~s}$; Ghosh \& Nelemans, 2015); other than being more sensitive, these telescopes operate as stand-alone facilities; therefore, a telescope like RAMSES could have a niche role alongside a more sensitive instrument. The wide FoV and rapid slew of RAMSES allows for rapid scanning of the GW error region for bright transients that can then be targeted for instantaneous deep follow-up by the Zadko telescope.

We plan to build and install RAPIDO (very rapid slewing mount co-located with Zadko) using two wide field telescopes combined with a CCD imager that will achieve $m<17$ with FoV (8-10) $\mathrm{deg}^{2}$. The single telescope system, comparable to RAMSES but using off the shelf optics, is expandable and can accommodate either $0.4 \mathrm{~m}$ or $2 \times 0.2 \mathrm{~m}$ telescopes on each mount. The system will provide low latency wide-field

\footnotetext{
${ }^{8}$ From the GW Candidate event Database, GraceDB; https://gracedb.ligo. org/

${ }^{9}$ http://allafrica.com/stories/201512040896.html

searches for transients and will automatically send alerts to Zadko for deep follow-up $(m<21)$ of any transient candidate. We note that when Virgo joins aLIGO, for the brightest GW sources the localisation error regions will reduce to some tens of degrees, providing opportunities for low-latency imaging of the entire field by RAPIDO and deep follow-up by Zadko.

\section{FAST RADIO BURST OPTICAL FOLLOW-UP}

The discovery of FRBs revealed the existence of an exciting new class of astronomical object (Lorimer et al., 2007). FRBs are a transient phenomenon of Jy level flux found in $\mathrm{GHz}$ bands with a very short duration of a few ms. FRBs are very difficult to localise, as they are mostly detected by single radio dishes (more than a dozen from Parkes and one from Arecibo) with FoVs of many arc-minutes. As of Feb 2016, no repeating events or counterparts in other wavelengths had been publicly announced Petroff et al. (e.g. for FRB140514 as reported in 2015). The first localisation, made public in Feb 2016 (FRB 150418; Keane et al., 2016) was achieved by the discovery of a fading radio transient, which enabled deep optical imaging to identify the host galaxy at $z \approx 0.5$. Identification of an afterglow and host was later called into question as the source was identified as an AGN (Williams \& Berger, 2016). Furthermore, observations of FRB 110523 using archival data from the Green Bank Telescope (Masui, 2015) favour models involving young stellar populations such as magnetars, over models involving the mergers of neutron stars, which are more likely to be located in low-density regions of the host galaxy. These contradictions can be resolved by obtaining multiple coincident multi-wavelength EM (especially optical) counterparts, and to search for possible $\mathrm{GW}$ signatures.

\subsection{Zadko Telescope automated follow-up of Parkes}

The Zadko Telescope and in the future RAPIDO telescopes, based on their reaction times and sensitivities for GRB imaging, have the potential to fill a niche role in extremely rapid follow up of radio transients events (see Figure 7). Previously, the most rapid follow up of FRB140514 was $7 \mathrm{~h}$ later, with ATCA radio telescope. The most rapid optical follow up was performed by GROND (GRB Optical/Near-Infrared Detector) but this was $16 \mathrm{~h}$ post-alert. SWOPE ${ }^{10}$ observations, also more than $16 \mathrm{~h}$ after the event, provided a limiting magnitude of only $R=16$. None of these follow-ups could offer a candidate for the host galaxy.

As of April 2015, the Zadko Telescope joined a large collaboration that is searching for FRBs in real time. The project is part of the alert network for the SUPERB ${ }^{11}$ and UMOST ${ }^{12}$ projects. As part of this effort the Zadko control software has

\footnotetext{
${ }^{10} \mathrm{http}: / /$ obs.carnegiescience.edu/swope

${ }^{11} \mathrm{https} / / /$ sites.google.com/site/publicsuperb/fast-radio-bursts

$12 \mathrm{http} / / /$ astronomy.swin.edu.au/research/utmost
} 


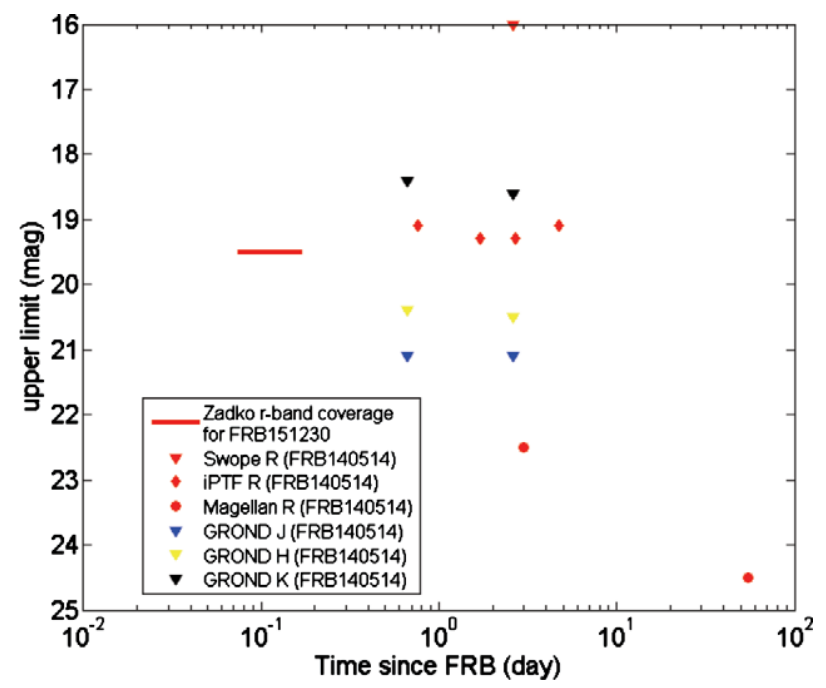

Figure 7. Zadko on source delay time (horizontal left line) for FRB followup, compared to all other optical follow-ups (symbols). The low latency FRB response was obtained during a Parkes shadowing experiment in December 2015, which set the record for the shortest time delay to image the FRB alert. The Zadko limiting magnitude of 19.5 (60 s exposure in $\mathrm{r}$ ) is improved by about $0.8 \mathrm{mag}$ in the stacked image. A complete analysis of this data will be presented in a subsequent work.

been updated to respond robotically to FRB alerts. Results from these observations have the potential to probe a region of parameter space not covered.

In December 2015, the team commenced a pilot project for several weeks that enabled the Zadko Telescope to shadow (follow) the same sky location as Parkes. Because Parke's beam is about four times the area of Zadko's FoV, we employed a tiling strategy. This first Zadko pilot experiment broke the record for the fastest response (about 40-min postburst), and earliest optical data following an FRB alert (see Figure 7). The first imaging of the source occurred before the human vetted alert was sent out from Parke's (analysis of this low latency data is on-going and will be reported in a subsequent work).

Based on event rate estimates from the 2015 observing sessions, 10 FRBs should be discovered in Parkes data in 2017. This provides an opportunity to search for a very early optical counterpart, to identify a possible short GRB afterglow, localise a host galaxy to confirm the distance, and to study the host environment.

\section{EDUCATION, OUTREACH, AND TRAINING}

\subsection{Science education enrichment}

The Zadko Telescope has been used for several novel science education programs. Local $\mathrm{PhD}$ student projects include the evaluation of authentic learning by participation in research projects (Heary et al., 2012; Coward et al., 2011). These projects include local school students identifying and searching for near Earth asteroids using the Zadko Telescope. This was extended as part of the Yachad visiting scholar award (led by Coward in November 2012) who initiated a pilot scheme that provided local school students in Israel access to the UWA Zadko Telescope for participating in the above project. In addition, there has been numerous undergraduate student training programs, both hosted locally and remotely from France that have used the Zadko Telescope for their projects.

\subsection{Space science training: space debris}

The potential exponential growth of artificial debris in Earth orbit together with its consequent hazard to satellite operations (Kennewell \& Brockman, 2007) has been known for some time (Kessler and Cours-Palais, 1978). Most observations and studies have concentrated on the low Earth orbital (LEO) regime. There are several military and civilian observing patrol networks that contribute to the maintenance of catalogues of the larger debris sizes $(>10 \mathrm{~cm})$. Occasional scientific campaigns are undertaken to explore smaller sized debris, as it has been realised that the growth of even micron sized debris has the potential to interfere with astronomical observations in the medium future (Biggs \& Kennewell, 2012).

Collisional velocities in geosynchronous Earth orbit (GEO) are lower than those in LEO, and this has led, along with the greater difficulty of observation, to a lesser interest in GEO debris. However, the discovery a few years ago of a previously unknown GEO debris population (Schildknecht et al., 2004; Schildknecht, Musci, \& Flohrer, 2007) has led to the realisation that the hazard in this region has been considerably underestimated. This high altitude region is totally beyond the range of radars used for LEO patrol, and metreclass optical instruments are required for useful analyses.

The Zadko telescope has previously been involved in imaging Australian space debris (Laas-Bourez, Kennewell, \& Coward, 2011), and we currently have an open $\mathrm{PhD}$ project to characterise the GEO debris population in this longitudinal region of the geosynchronous orbit. (One of us has been involved in a study with Kitt Peak National Observatory that has shown that the large space object population at GEO shows a significant longitudinal variation.)

The substantial public and schools interest in artificial space debris (space debris is a component of the WA school science curriculum) offers a great opportunity for public outreach in this field as well as for training of teachers and students in the acquisition of hands on skills in planning and implementing telescope scheduling and debris database access and analysis. These activities are expected to be coordinated with a co-located node of the Falcon Telescope Network dedicated space surveillance facility (Chun et al. 2014).

\section{SUMMARY AND EMERGING PRIORITIES}

The Zadko Telescope has been operating as a fully robotic optical facility prioritised for automated transient source photometry from March 2010. From 2012 to 214, the facility 
underwent a major upgrade with the installation and commissioning of an automated observatory building. Despite lengthy disruptions to the operation during this period, the Zadko Telescope emerged as the premier Australian facility for automated triggered follow-up of gamma bursts (40 follow-ups) and neutrino localisations (58 follow-ups). In the context of GRBs, the Zadko Telescope has placed constraints on a possible GRB origin for two neutrino events that were rapidly followed up (ANT140301A and ANT141220A).

Solar System science has been an important component of the overall Zadko science program. From 2014 to 2015, the telescope contributed to accurate period determinations for five asteroids by obtaining critical light curve measurements. The accuracy of relative photometry by Zadko exceeded the project requirements, i.e. dispersion $<0.01$ magnitudes for targets of relatively high brightness ( $\mathrm{V} \sim 11-13)$, despite the poor seeing at the site.

Although GRB and neutrino follow-up will continue to be a priority for the transient source program, there are at least two emerging fields that will become a priority for the facility. Rapid follow-up of FRB triggers from Parkes has the potential for breakthrough science, given the debate on the origin of FRBs. This first Zadko pilot experiment broke the record for the fastest response and earliest photometric limits from an FRB alert (Figure 7). The first imaging of the source occurred before the human vetted alert was sent from Parkes (analysis of this low latency data will be presented in a later study).

Optical follow-ups of GW alerts from aLIGO have the potential to unveil the physics of neutron star mergers. Given the poor localisation of aLIGO (which will improve when joined by Virgo), we are planning an upgrade to the Zadko facility. It will consist of co-locating a very rapid response wide FoV two telescope system that will be used to scan much of the GW error region. Transient candidates identified by the wide FoV system will be automatically, and rapidly followed up with deep imaging by the Zadko Telescope. When Virgo joins aLIGO are at design sensitivity, the brightest GW sources will be localised to some tens of degrees, allowing low-latency imaging of the entire field by the RAPIDO Zadko facility.

\section{ACKNOWLEDGEMENTS}

The Zadko Telescope was made possible by a philanthropic donation by James Zadko, Director of Clair Energy, to the UWA. In addition, we thank the West Australian Government (Department of Environment and Conservation), Perth Observatory, Australian Research Council grant (LE12010051), and the University of Western Australia for financial support for the upgraded Zadko Observatory. D.M. Coward was supported by the Australian Research Council Future Fellowship (2011-2015 FT100100345) and acknowledges advice from S. Driver and G. Meurer at the International Center for Radio Astronomy Research at UWA. B. Gendre acknowledges financial support from the NASA Award NNX13AD28A and the NASA Award NNX15AP95A. EJH acknowledges support from a UWA Research Fellowship. We also thank Andrew Burrell (School of Physics UWA), and France Romain Laugier for technical support and finding solutions to the many technical problems.

\section{REFERENCES}

Abadie, J., et al. 2010, CQGra, 27, 173001

Abbott, B. P., et al. 2016a, PhRvL, 116, 241103

Abbott, B. P., et al. 2016b, ApJL, 826, L13

Abbott, B. P., et al. 2016c, PhRvL, 116, 061102

Acernese, F., et al. 2015, CQGra, 32, 024001

Ageron, M., et al. 2011, NIMPA, 656, 11

Ageron, M., et al. 2012, APh, 35, 530

Acharya, B. S., et al. 2013, APh, 43, 3

Barthelmy, S. D., et al. 1994, in AIP Conf. Proc., 307, 643

Bellm, E. 2014, in Proc. of The Third Hot-Wiring the Transient Universe Workshop, P. R. Wozniak, M. J. Graham, A. A Mahabal, \& R. Seaman R., eds. (held in Santa Fe, November 13-15), 27

Berger, E., Fong, W., \& Chornock, R. 2013, ApJL, 774, L23

Biggs, J., \& Kennewell, J. 2012, Orbital Space Debris and Sky Brightness, in Proc. of the 11th Australian Space Science Conference (Sydney: National Space Society of Australia Ltd.), 157

Boër, M., Gendre, B., \& Stratta, G. 2015, ApJ, 800, 16

Bromm, V., \& Loeb, A. 2006, ApJ, 642, 382

Carry, B. 2012, P1Sci, 73, 98

Cellino, A., Bagnulo, S., Tanga, P., Novaković, B., \& Delbo, M. 2014, MNRAS, 439, L75

Cellino, A., Belskaya, I. N., Bendjoya, Ph., Di Martino, M., GilHutton, R., Muinonen, K., \& Tedesco, E. F. 2006, Icarus, 180, 565

Chun, F., Tippets, R., Dearborn, M., Gresham, K., Freckleton, R., \& Douglas, M. 2014, in Proc. of the 2014 AMOS Technical Conference, S. Ryan, ed. (held in Maui, September 9-12), E64

Connaughton, V., et al. 2016, ApJL, 826, L6

Collaboration, T. L. S., et al. 2015, CQGra, 32, 074001

Coward, D. M., et al. 2010, PASA, 27, 331

Coward, D. M., et al. 2011, AdSpR, 47, 1922

Delbo, M., Ligori, S., Matter, A., Cellino, A., \& Berthier, J. 2009, ApJ, 694, 1228

Devogèle, M., et al. 2015, MNRAS, 453, 2232

Dubus, G., et al. 2013, APh, 43, 317

Durech, J., Sidorin, V., \& Kaasalainen, M. 2010, 513, A46

Eichler, D., et al. 1989, Nature, 340, 126

Galama, T. J., et al. 1998, Nature, 395, 670

Gehrels, N., et al. 2004, ApJ, 611, 1005

Gehrels, N., et al. 2008, ApJ, 689, 1161

Gendre, B., et al. 2011, A\&A, 530, 74

Gendre, B., et al. 2012, ApJ, 748, 59

Gendre, B., et al. 2013, ApJ, 766, 30

Ghosh, S., \& Nelemans, G. 2015, Ap\&SS, 40, 51

Gil-Hutton, R., Mesa, V., Cellino, A., Bendjoya, P., Pealoza, L., \& Lovos, F. 2008, AstAp, 482, 309

Hamuy, M., \& Suntzeff, N. B. 1990, AJ, 99, 1146

Harris, A. W., Warner, B. D., \& Pravec, P., eds. 2012, Asteroid Lightcurve Derived Data V13.0 (NASA Planetary Data System)

Heary, A., Coward, D., Blair, D., \& Venville, G. 2012, in 39th COSPAR Scientific Assembly (held in Mysore, July 14-22), 39, 735

Howell, E. J., et al. 2015, PASA, 32, 46

IceCube Collaboration et al. 2006, APh, 26, 155

Jakobsson, P., et al. 2004, ApJ, 617, L21 
Janka, H. T., Hanke, F., Hüdepohl, L., Marek, A., Müller, B., \& Obergaulinger, M. 2012, PTEP, submitted, arXiv:1211.1378

Johansen, A., \& Klahr, H. 2011, EM\&P, 108, 3943

Kann, D. A., et al. 2010, ApJ, 720, 1513

Kanner, J., et al. 2008, CQGra, 25, 184034

Keane, E. F., \& Petroff, E. 2015, MNRAS, 447, 2852

Keane, E. F., et al. 2016, Nature, 530, 453

Keller, S. C., et al. 2007, PASA, 24, 1

Kennewell, J., \& Brockman, A. 2007, in Proc. of the 7th Australian Space Science Conference (Sydney: National Space Society of Australia Ltd.), 139

Klotz, A., Boër, M., Atteia, J. L., \& Gendre, B. 2009, AJ, 137, 4100

Kouveliotou, C., Meegan, C. A., Fishman, G. J., \& Bhat, N. P. 1993, ApJ, 413, L101

Laas-Bourez, M., Kennewell, J., \& Coward, D. 2011, in Proc. of the 10th Australian Space Science Conference (Sydney: National Space Society of Australia Ltd.), 514

Lennarz, D., et al. 2013, in Proc. of 7th Huntsville GammaRay Burst Symposium (held in Nashville, April 14-18), C1304143

Li, L.-X., \& Paczyński, B. 1998, ApJL, 507, L59

Lorimer, D. R., Bailes, M., McLaughlin, M. A., Narkevic, D. J., \& Crawford, F. 2007, Science, 318, 777

MacPherson, D., Coward, D. M., \& Zadnik, M. G. 2013, ApJ, 779, 73

Maoz, D., Mannucci, F., \& Nelemans, G. 2014, ARAA, 52, 107

Masiero, J., \& Cellino, A. 2009, Icarus, 199, 333

Masui, K., et al. 2015, Nature, 528, 523

Meszaros, P. 2006, RPPh 69, 2259

Mészáros, P., \& Rees, M. J. 1997, ApJ, 476, 232

Metzger, B. D., et al. 2010, MNRAS, 406, 2650

Modjaz, M., et al. 2009, ApJ, 702, 226

Morales-Luis, A. B., Sanchez Almeida, J., Aguerri J. A. L., \& Munoz-Tunon, C. 2011, ApJ743, 77

Mousis, O., et al. 2014, ExA, 38, 91

Murphy, T., et al. 2013, PASA, 30, 6

Nakauchi, D., Suwa, Y., Sakamoto, T., Kashiyama, K., \& Nakamura, T. 2012, ApJ, 759, 128

Nomoto, K. 1982, ApJ, 253, 798

Panaitescu, A., Mészáros, P., \& Rees, M. J. 1998, ApJ, 503, 314

Perlmutter, S., et al. 1999, ApJ, 517, 565

Petroff, E., et al. 2015, MNRAS, 447, 246

Rees, M. J., \& Mészáros, P. 1992, MNRAS, 258, 41
Richmond, M. W., Treffers, R. R., Filippenko, A. V., Paik, Y., Leibundgut, B., Schulman, E., \& Cox, C. V. 1994, AJ, 107, 1022

Riess, A. G., et al. 1998, AJ, 116, 1009

Rhoads, J. E. 1999, ApJ, 525, 737

Rosswog, S. 2005, ApJ, 634, 1202

Schildknecht, T., Musci, R., \& Flohrer, T. 2007, in Proc. of the 2007 AMOS Technical Conference (held in Wailea, September 12-15), 354

Schildknecht, T., et al. 2004, AdSpR, 34, 901

Shawhan, P. S. 2012, in SPIE Conf. Ser., Vol. 8448, Observatory Operations: Strategies, Processes, and Systems IV, ed. A. B. Peck, R. L. Seaman, \& F. Comeron (Bellingham: SPIE), 84480Q

Singer, L. P., et al. 2014, ApJ, 795, 105

Soderberg, A. M., et al. 2008, Nature, 453, 469

Stritzinger, M., et al. 2002, AJ, 124, 2100

Sunshine, J., Connolly, H. C., McCoy, T. J., Bus, S. J., \& La Croix, L. M. 2008, Science, 320, 514

Suwa, Y., \& Ioka, K. 2011, ApJ726, 107

Tanga, P., et al. 2015, MNRAS, 448, 3382

Tanvir, N. R., Levan, A. J., Fruchter, A. S., Hjorth, J., Hounsell, R. A., Wiersema, K., \& Tunnicliffe, R. L. 2013, Nature, 500, 547

The LIGO Scientific Collaboration et al. 2016, Phys. Rev. X, 6, 041015

Tingay, S. J., et al. 2013, PASA, 30, 7

Tinney, C. G., Ryder, S. D., Ellis, S. C., Churilov, V., Dawson, J., Smith, G., Waller, L., \& Whittard, J. 2004, in SPIE Conf. Ser., Vol. 5492, Astronomical Telescopes and Instrumentation (Bellingham: SPIE) 998

Todd, M., Coward, D. M., Tanga, P., \& Thuillot, W. 2013, PASA, 30,14

Todd, M., Tanga, P., Coward, D. M., \& Zadnik, M. G. 2012a, MNRAS, 420, L28

Todd, M., Tanga, P., Coward, D. M., \& Zadnik, M. G. 2012b, MNRAS, 424, 372

Tornatore, L., Ferrara, A., \& Schneider, R. 2007, MNRAS, 382, 945

Whelan, J., \& Iben, I. 1973, ApJ, 186, 1007

Williams, P. K. G., \& Berger, E. 2016, ApJL, 821, L22

Williams, R. D., Barthelmy, S. D., Denny, R. B., Graham, M. J., \& Swinbank, J. 2012, in SPIE Conf. Ser., Vol. 8448, Observatory Operations: Strategies, Processes, and Systems IV, ed. A. B. Peck, R. L. Seaman, \& F. Comeron (Bellingham: SPIE), 84480R Willingale, R., et al. 2007, ApJ, 662, 1093

Woosley, S. E. 1993, ApJ, 405, 273 